\title{
Formación en Psicología Comunitaria en una Universidad Chilena: Sistematización de un Trabajo Territorial con Migrantes
}

\section{Training in Community Psychology at a Chilean University: Systematization of Territorial Work with Migrants}

\author{
Paula Riveros Ahumada y Rubén Nilo Pérez \\ Universidad Santo Tomás
}

Bárbara Olivares Espinoza
Universidad Diego Portales

\author{
María Isabel Reyes Espejo \\ Pontificia Universidad Católica de Valparaíso
}

\begin{abstract}
Se presentan los resultados de una experiencia de formación académica en psicología comunitaria, en desarrollo desde el año 2013 en una universidad privada chilena ubicada en la ciudad de Santiago. Dicha experiencia, en curso, se ha implementado mediante la vinculación institucional con el municipio de Independencia (área urbana de Santiago) y se ha orientado hacia el trabajo con la población migrante crecientemente avecindada en dicha localidad. La experiencia se describe tanto en base al marco institucional posibilitado por la política de aprendizaje y servicio de la universidad, como por la propuesta pedagógica asumida por el equipo docente y la evaluación de los actores locales involucrados. La descripción de esta propuesta, se realiza a través de la revisión y análisis documental de información proveniente de diversas fuentes escritas, 8 entrevistas individuales a miembros de la comunidad y funcionarios municipales, 2 grupos focales a estudiantes y 18 cuestionarios aplicados a docentes y estudiantes, mediante un muestreo intencionado. La información es analizada cualitativamente, mediante la técnica de análisis temático. Los resultados describen el desarrollo del proyecto formativo en un marco institucional definido desde una política pública que aprovecha el contexto de migración para liderar acciones de intervención en el ámbito local, atendiendo a las demandas sociales vinculadas al fenómeno.
\end{abstract}

Palabras clave: formación, migración, psicología comunitaria

\begin{abstract}
This article presents the results of an academic training experience in Community Psychology that has been ongoing since 2013 at a Chilean private university located in the city of Santiago. This ongoing experience was implemented through an institutional agreement with the Independencia municipality (urban area of Santiago) and has focused on the increasingly large migrant population present in this district. The experience is described considering the institutional framework made possible by the learning and service policy of the university, the educational approach adopted by the teaching team, and the assessment of all the local actors involved. This proposal is described through the documentary review and analysis of several written sources, 8 individual interviews with community members and municipality workers, 2 focus groups with students, and 14 surveys administered to teachers and students. Purposive sampling was used. The information is analyzed qualitatively using thematic analysis. Results describe the development of the training project within an institutional framework defined through a public policy that takes advantage of the migration context to implement interventions at a local level, thus responding to the social demands derived from the phenomenon.
\end{abstract}

Keywords: training, migration, community psychology

A partir de la publicación de la Ley General de Universidades del año 1981, el número de universidades que imparten la carrera de psicología en Chile ha aumentado progresivamente, ofreciendo formación tanto a nivel de pre como de posgrado. En este contexto y desde los años 90, la psicología comunitaria (en adelante PC) chilena, ha experimentado un desarrollo significativo en el ámbito académico, siendo progresivamente incorporada en las mallas curriculares de pregrado en psicología (Asún, 2005; Krause, Jaramillo Torrens, Monreal, Carvacho García \& Torres, 2011; Rozas \& Martínez, 2007). Actualmente, existe un total de 60 universidades en Chile, de las cuales 51 dictan la carrera de psicología (43 acreditadas). La acreditación es un proceso voluntario al que se someten las instituciones de educación

Paula Riveros Ahumada y Rubén Nilo Pérez, Escuela de Psicología, Universidad Santo Tomás, Santiago, Chile; Bárbara Olivares Espinoza, Facultad de Psicología, Universidad Diego Portales, Santiago, Chile; María Isabel Reyes Espejo, Escuela de Psicología, Pontificia Universidad Católica de Valparaíso, Chile.

La correspondencia relativa a este artículo debe ser dirigida a Paula Riveros Ahumada, Facultad de Ciencias Sociales y Comunicaciones, Escuela de Psicología Universidad Santo Tomás, Dirección Ejército 146, Santiago, Chile. E-mail: paulariveros@santotomas.cl 
superior autónomas, así como las carreras de pregrado, programas de posgrado y especialidades del área de la salud que ellas imparten, para contar con una certificación de calidad de sus procesos internos y resultados. La acreditación de carreras y programas certifica la calidad en función de sus propósitos declarados y de los criterios establecidos por las respectivas comunidades académicas y profesionales (Comisión Nacional de Acreditación-Chile [CNA], 2013). De estas acreditadas, 34 cuentan con uno o más cursos de PC y ocho imparten la especialidad como una salida profesional (Olivares, Reyes, Berroeta \& Winkler, 2016). En las mallas curriculares, esto se traduce en asignaturas que reciben el nombre de "Psicología Comunitaria", "Problemas Psicosociales", "Intervención Social o Psicosocial" o "Salud Mental Comunitaria", existiendo también una inclusión transversal que considera la perspectiva comunitaria en diversas asignaturas de la carrera (Olivares et al., 2016).

A pesar de la sostenida y creciente implementación de cursos de PC en la formación de pregrado en psicología durante los últimos 10 años, esta formación disciplinar continúa teniendo un carácter eminentemente teórico, una implementación curricular inorgánica y secundaria a las especialidades tradicionales, así como importantes limitaciones institucionales para su aplicación práctica en las comunidades y territorios. En este contexto, la introducción de la metodología aprendizaje-servicio (A+S) en Latinoamérica y en algunos países de Europa ha permitido avanzar en la generación de propuestas formativas que dialogan con las necesidades comunitarias y que permiten formar a profesionales sensibles con un profundo compromiso ciudadano por la transformación del entorno, fomentando una ciudadanía activa, responsable y cohesionada (Tapia, 2006). Teniendo como marco general el A+S, se ha propuesto el desarrollo de una experiencia pedagógica que articula procesos de intervención comunitaria con la formación de estudiantes en el campo de la PC, para dar respuesta a necesidades específicas vinculadas al ámbito de la migración.

La experiencia desarrollada es relevante, sobre todo cuando se considera el lugar subalterno de la PC en la formación universitaria de psicología en Chile (Olivares et al., 2016), en un contexto social caracterizado por la implementación sistemática y hegemónica del neoliberalismo en todas las esferas de la vida cotidiana. Para una disciplina que se ha desarrollado y definido en torno al principio de la inseparabilidad entre teoría y práctica, estas dificultades no son menores. Por ello, parece relevante explorar alternativas que abran ciertas pistas para la formación en PC en este contexto, formulando, entonces, la siguiente pregunta: ¿Cómo es posible implementar una propuesta de formación en PC que sea consistente y coherente con sus principios a la base, en un contexto social marcadamente neoliberal que propicia el individualismo y la competitividad, tanto en el ámbito universitario como en los propios territorios y comunidades?

Para responder a esta pregunta, este artículo busca describir y analizar la implementación de una experiencia de formación universitaria en PC, que se ha desarrollado desde el año 2013 en alianza con la Oficina de Migración de la Municipalidad de Independencia, en pleno centro urbano de la ciudad de Santiago. Conocer esta experiencia de vinculación directa de los estudiantes con los territorios y vecinos, nacionales e inmigrantes, y con sus múltiples problemáticas emergentes, resulta particularmente relevante para pensar la formación actual en psicología. Al igual que le ha sucedido a los gobiernos locales y los programas sociales, el impacto psicosocial de la inmigración en los últimos 10 años ha exigido que las universidades se involucren en el abordaje de un problema para el que no estaban preparadas. Cabe, entonces, la responsabilidad de generar y compartir experiencias y conocimientos locales situados, para acercar el ejercicio profesional a las necesidades cambiantes de las poblaciones.

En este artículo se describe el proceso de implementación de una propuesta de formación en PC que se desarrolló en un ámbito local particular, donde la migración opera como telón de fondo, rescatando los discursos de los principales actores involucrados en la experiencia: estudiantes, docentes y participantes. Con ello, se buscó visibilizar el aporte de la PC chilena en el continuo formación-intervención/investigación, constituyéndose en una experiencia que logra articular procesos de intervención psicosocial en comunidades y territorios concretos, permitiendo superar las tradicionales limitaciones de las intervenciones esporádicas y descontextualizadas que han caracterizado la formación de pregrado en psicología (Olivares et al., 2016).

\section{Formación Profesional en Psicología Comunitaria}

$\mathrm{Al}$ referirse al concepto de formación profesional, se asume que "no es un fin en sí misma, sino un medio para desarrollar las aptitudes profesionales de una persona, teniendo en cuenta las posibilidades de empleo y de permitirle hacer uso de sus capacidades como mejor convenga a sus intereses y a los de la comunidad" 
(Casanova, 2003). En particular, para la formación en PC es necesario reconocer a los/as psicólogos/as ${ }^{1}$ comunitarios como agentes de cambio social, con compromiso ético y político con las comunidades, críticos, que utilizan herramientas para facilitar la participación ciudadana, la autogestión y el fortalecimiento de las comunidades (Rodríguez Kauth, 2008). Así, la formación en PC requiere de un esfuerzo permanente de monitoreo crítico que invite a los docentes a desarrollar prácticas pre-profesionales consistentes con los planteamientos de la propia disciplina (Winkler, Alvear, Olivares \& Pasmanik, 2014).

Por otro lado, Prilleltensky (2001) señala que los estudiantes de esta área están preparados con metodologías de intervención e investigación; no obstante, no hay un cuestionamiento de los valores involucrados en las mismas. El accionar de la PC estaría en estas condiciones, orientado al bienestar individual y no a la relación del individuo en comunidad. En este sentido, académicos y estudiantes estarían centrando sus prácticas en uno u otro sentido, sin integrar ni cumpliendo la función de traducir la reflexión ética y la investigación a acciones sociales. Por lo mismo, una visión que considere el contexto es necesaria, ya que aporta dimensiones que en otros enfoques de la psicología no son contemplados (Riveros, 2012; Winkler, Pasmanik, Alvear \& Olivares, 2015).

En otro plano, el contexto en el que se desarrolla el trabajo comunitario en la actualidad se ha visto cuestionado desde la llamada institucionalización de la PC, que la ha puesto como referente en las prácticas institucionales de intervención psicosocial (Castillo \& Winkler, 2010), trayendo, como consecuencia, el abandono de algunos principios que fundaron la disciplina y, por consiguiente, la casi nula participación de los actores involucrados en los programas orientados hacia la resolución de sus problemas (Reyes Espejo, Olivares Espinoza, Berroeta Torres \& Winkler Müller, 2015). Este panorama plantea, entonces, una preocupación a la hora de definir qué temas son prioritarios al momento de pensar la formación, siendo necesario preguntarse si estaría respondiendo al escenario concreto de trabajo para los profesionales o si se están discutiendo tópicos provenientes de una noción ideal de PC que se mantienen en un plano teórico sin avanzar en desarrollos aplicados (Riveros, 2012).

Coincidiendo con los planteamientos de Montero y Giuliani (1997), resulta relevante proponer nuevas metodologías en la formación de los psicólogos comunitarios, que permitan generar prácticas coherentes con los principios y valores de la disciplina. Es así como la brecha entre lo que se enseña y lo que realmente se necesita saber ha motivado a la constitución de nuevas formas, métodos, estrategias y sistemas de formación para enseñar lo que demanda la realidad laboral (Winkler et al., 2015; Yañez Canal, 2005). Por tanto, es clave estudiar y trabajar la relación universidad-comunidad en lo conceptual y práctico, con el objetivo de reflexionar en torno a la formación y adecuación de las prácticas preprofesionales a los contextos actuales (Montero \& Serrano-García, 2011).

Es en el marco de esta relación, que a partir de 2013, se inicia un proceso de instalación territorial de la línea comunitaria de una escuela de psicología de una universidad privada, ubicada en Santiago (Región Metropolitana) que permite impulsar un proceso formativo que dialoga estrechamente con la realidad local y, desde allí, se retroalimentan contenidos y metodologías. A nivel territorial, las comunidades se ven enfrentadas a múltiples problemáticas sociales difícilmente abordables con los marcos de referencia construidos por la disciplina en los últimos 20 años.

Específicamente, una de las problemáticas sociales que viene interpelando de manera creciente a la sociedad chilena en el último tiempo, es la migración que no solo pone en entredicho a la propia disciplina, sino también a las instituciones y a la sociedad en general. Surgen así, demandas concretas que se acentúan, al no existir políticas públicas que presten apoyo a la población migrante que llega y se asienta en el país.

Consistentemente, en el contexto internacional, desde el nacimiento del nuevo milenio, ha surgido una serie de propuestas formativas a nivel universitario que vinculan los tema de ciudadanía y responsabilidad social, traducidos en planteamientos que abogan por una formación en valores y donde se cruzan contenidos en el marco de una ciudadanía intercultural (Bilbeny, 2004; Bolívar, 2005; Cortina, 2001; Martínez, Buxarrais \& Bara, 2002). La condición actual de Chile como país en vías de desarrollo, insertándose en los flujos de la economía global (Sassen, 2003) y situándose también como un país receptor de migración

\footnotetext{
${ }_{1}^{1}$ Para facilitar la lectura del texto, se obviará en adelante la distinción lingüística de género, pese a que las/los autoras/es asumen la importancia de dicho enfoque.
} 
relevante en el cono sur, revela el carácter diferenciador y particular que puede adquirir esta realidad, en comparación con otras latitudes del continente.

\section{Migración en Chile}

Chile ha pasado de ser un país eminentemente generador de emigrantes a recibir en las últimas dos décadas una mayor cantidad de ciudadanos extranjeros. En lo concreto, el número de inmigrantes en el país ha crecido exponencialmente en los últimos años, alcanzando el 6,6\% de la población total (Instituto Nacional de Estadísticas-Departamento de Extranjería y Migración, INE-DEM, 2019). La gran mayoría corresponde a inmigración de origen latinoamericano, siendo Venezuela (288.233), Perú (223.923), Haití (179.338), Colombia (146.582) y Bolivia (107.346) los países de origen que concentran la mayor cantidad de inmigrantes (INE-DEM, 2019).

El aumento sostenido de población inmigrante en Chile ha generado, como efecto, una creciente visibilidad pública del fenómeno que, sin embargo, ha contribuido a la construcción de una percepción de la inmigración como un problema social. Esta percepción, fuertemente alimentada por los medios de comunicación y observable en las relaciones cotidianas, genera prejuicios en torno a los migrantes, responsabilizándolos de las tasas de desempleo, la falta de disponibilidad de servicios públicos para la población nacional y el aumento de la delincuencia (Tijoux \& Palominos Mandiola, 2015). Se produce, de este modo, una serie de dificultades que se expresan en la cotidianidad de la vida social, repercutiendo en la generación de prácticas discriminatorias que afectan la convivencia cotidiana en los barrios.

\section{Método}

Para describir y analizar la experiencia de formación en PC en un contexto local particular, se optó por una metodología cualitativa, considerada la más adecuada cuando se asume la necesidad de describir y comprender realidades que se transforman y construyen dinámicamente en relación a las lógicas contextuales y a la interpretación que dan los propios actores desde los lugares en que se encuentran. También implica la valoración de la diversidad, del contexto y la importancia de los sujetos sociales, en referencia a su discurso y accionar (Banyard \& Miller, 1998; Mieles Barrera, Tonon \& Alvarado Salgado, 2012; Stein \& Mankowski, 2004).

Dentro del encuadre cualitativo, se recurrió a la sistematización de una experiencia educativa, entendiéndola como un sistema de acción, donde diferentes actores construyen y reproducen sentidos desde sus propios horizontes culturales y sociales (Martinic, 2010). La sistematización se constituyó, entonces, en un proceso metodológico de reflexión y de producción de conocimientos de las experiencias prácticas, donde se construyó un lenguaje descriptivo propio, "desde adentro" de las propias experiencias, reconstituyendo y explicitando el marco de referencia que le da sentido (Martinic, 2010).

\section{Participantes}

Los participantes del estudio fueron 18 estudiantes, 6 docentes, 3 funcionarios municipales y 5 miembros de la comunidad. Se seleccionaron estudiantes que cursaron las asignaturas descritas en el plan curricular del área psicología social comunitaria y docentes que realizaron estas asignaturas. Los funcionarios municipales seleccionados corresponden a aquellos que realizaron labores de coordinación y articulación de intervenciones derivadas de las asignaturas descritas. Los miembros de la comunidad corresponden a aquellos actores sociales locales, dirigentes y miembros de organizaciones que participaron de las intervenciones en la comuna de Independencia. El tipo de muestreo realizado fue intencional por conveniencia. A los estudiantes se les convocó a un grupo focal, una vez egresados de las asignaturas del área social comunitaria, a través de una invitación dirigida individualmente y firmada por la coordinación del área, mientras que a los docentes, se les hizo llegar un correo electrónico, una vez finalizado el curso que dictaron, solicitando colaborar con procesos de retroalimentación, que forman parte de la estrategia de vinculación con el medio de la carrera. A los funcionarios municipales y a los dirigentes locales, se les visitó en sus lugares de trabajo, y se solicitó su participación como parte de los procesos de evaluación y monitoreo de las acciones desarrolladas en alianza con el gobierno local de Independencia. Dichas acciones acostumbran ser evaluadas bilateralmente, al principio y al término de cada semestre académico. 


\section{Técnicas de Producción de Información}

La producción de información se canalizó a través de diversas técnicas:

Análisis documental y textual. Mediante este, se buscó acceder a los sentidos y significados provenientes del discurso institucional-académico respecto de la formación profesional en la actualidad. Esta técnica permite analizar todo tipo de documentos como productos habituales de los distintos contextos sociales que constituyen focos de interés para los investigadores cualitativos (Dulzaides Iglesias \& Molina Gómez, 2004; Íñiguez Rueda, 1999). Los documentos secundarios o complementarios poseen un carácter público o privado y contienen información relevante sobre el problema estudiado (Montero, 2006). Los documentos analizados se detallan en la Tabla 1.

Tabla 1

Documentos Analizados

\begin{tabular}{cll}
\hline Año & \multicolumn{1}{c}{ Autor } & \multicolumn{1}{c}{ Documento } \\
\hline 2013 & CNA & Aprueba reglamentos sobre áreas de acreditación acorde al artículo $17^{\circ}$ de la Ley 20.129 \\
2011 & Universidad & Programa de asignatura: Fundamentos Teóricos de la PC \\
2011 & Universidad & Programa de asignatura: Diagnóstico Social Comunitario \\
2011 & Universidad & Programa de asignatura: Evaluación de Proyectos Sociales \\
2011 & Universidad & Programas de asignatura: Intervención Comunitaria I y II \\
2015 & Universidad & Programa de asignatura: Electivo Migración \\
\hline
\end{tabular}

Grupo Focal. Se utilizó para obtener la perspectiva de los estudiantes. Tiene la forma de una entrevista grupal, en la que existen preguntas que guían la conversación, con la finalidad de que emerja una representación o comprensión del sujeto, respecto a la formación profesional, es decir, acceder a un sujeto en situación (Canales Cerón, 2006). Se efectuaron dos grupos focales. Algunas de las preguntas realizadas fueron: “Podrían describirnos la experiencia de trabajo realizada en la asignatura Intervención Social Comunitaria I y II?”; “Qué significó en términos de su formación el trabajo con chilenos/as y personas migradas?”; “Cuáles fueron los aprendizajes personales y profesionales?”.

Cuestionario. Estuvo conformado por preguntas cerradas y abiertas (Canales Cerón, 2006), dirigido a estudiantes y docentes que pertenecen al área social comunitaria de la universidad (participantes de los cursos respectivos), con la finalidad de profundizar en sus percepciones respecto del trabajo realizado en terreno. Se aplicaron 18 cuestionarios. Algunas de las preguntas cerradas planteadas a docentes fueron: "Las actividades contribuyeron positivamente a los procesos de enseñanza-aprendizaje de los estudiantes"; "Las actividades me permitieron generar nuevas formas de relación con el entorno"; "El desarrollo de las actividades me ha hecho reflexionar sobre la relevancia social de lo que hacemos". Por su parte, algunas de las preguntas cerradas planteadas a estudiantes fueron las siguientes: "Entiendo claramente la relación entre el contenido curricular de la asignatura y las actividades llevadas a cabo"; "La actividad me sirvió para aprender cosas que no se pueden aprender en la sala de clases."; "Mi participación en las actividades me ha hecho reflexionar sobre nuestra misión ciudadana, tanto como estudiantes así como futuros profesionales". Las preguntas abiertas formuladas a docentes fueron las siguientes: "Describa su experiencia docente en la asignatura de Intervención Social Comunitaria I y II"; "Mencione qué cambios debió realizar en su labor docente”.

Entrevista semiestructurada. Se aplicó para acceder a los sentidos y significados de los actores locales (funcionarios municipales) y de los participantes de las intervenciones (miembros de las comunidades que recibieron las intervenciones que acá se analizan). Se realizaron 8 entrevistas, algunas de las preguntas realizadas a los funcionarios municipales fueron: “¿Qué valora de la participación de la universidad en la generación de acciones locales?"; “¿Qué debería fortalecerse en la formación profesional para quienes trabajen en migrantes?”. A su vez, para los actores comunitarios, las preguntas fueron: “¿Cómo fue su experiencia al trabajar con estudiantes de psicología?”; “¿qué destacaría de esa participación?"; "Si pudiera sugerir cambios para que mejoren su trabajo, ¿qué les diría?”. 


\section{Procedimiento}

A continuación, se describe cronológicamente el procedimiento de recolección de información. Quienes realizaron este trabajo tenían la experiencia requerida para este tipo de procedimientos por su trayectoria en equipos de investigación.

1. Estudiantes: los grupos focales y cuestionarios fueron aplicados a estudiantes de psicología en dependencias de la universidad. Este trabajo fue realizado por académicos del área comunitaria que no estuvieron a cargo de los procesos de intervención desarrollados.

2. Docentes: los cuestionarios fueron aplicados a los docentes que estuvieron directamente a cargo de los procesos de intervención comunitaria desarrollados con las comunidades. Esta labor fue realizada por una ayudante de investigación en la misma universidad.

3. Funcionarios municipales: las entrevistas individuales se realizaron en las dependencias municipales en donde trabajan cotidianamente, y estuvieron a cargo de una ayudante de investigación.

4. Actores comunitarios: las entrevistas individuales con miembros de la comunidad estuvieron a cargo de un docente del área comunitaria y se desarrollaron tanto en el domicilio personal de los entrevistados como en sedes comunitarias en las que ellos participan.

Cabe señalar que todos los participantes del proceso de recolección fueron debidamente informados del objetivo y procedimientos del estudio y entregaron por escrito su consentimiento informado para su participación.

\section{Análisis de la Información}

La información fue sistematizada mediante la técnica de análisis temático, la que permitió identificar, organizar, analizar y reportar patrones o temas a partir de una cuidadosa lectura y relectura de la información recogida (Mieles Barrera et al., 2012) para inferir resultados que propicien una adecuada comprensión/interpretación del fenómeno en estudio. Son esenciales en este proceso: la familiarización con los datos; la generación de categorías o códigos iniciales y la búsqueda, revisión y definición de temas (Martínez Carazo, 2006; Vietyes, 2009).

\section{Resultados}

Los resultados que se presentan a continuación se organizan en tres ejes temáticos que dan cuenta del énfasis central en la caracterización de una propuesta pedagógica y metodológica, por un lado, y la definición de los componentes de la formación en PC, por otro.

\section{Propuesta Pedagógica}

Aprendizaje y servicio. Los planes de estudio de las asignaturas del área comunitaria contemplan las llamadas actividades de terreno, actividades prácticas que plantean la prestación de servicios bajo supervisión de un docente, proceso que facilita la adquisición de competencias profesionales y el desarrollo de valores, ya que el estudiante utiliza sus conocimientos y habilidades adquiridos en clases y se compromete emocionalmente con el sujeto con el que trabaja.

El estudiante da respuestas a demandas locales que requieren de apoyo técnico para la implementación de procesos diagnósticos y de acción comunitaria en ámbitos de interés para los gobiernos locales. En dichos procesos, se:

prioriza un enfoque participativo para el diagnóstico e intervención en la comunidad, enfatizando la evaluación crítica para la intervención, identificación, técnicas de recolección y análisis de la información. (Programa curso Diagnóstico Social Comunitario, 2011)

Además:

[la asignatura] pretende analizar y aplicar diversas metodologías que permitan el monitoreo y evaluación de proyectos sociales. (Programa curso Evaluación de Proyectos Sociales, 2011) 
Así, también, y luego de iniciar esta relación de colaboración, el equipo docente se sitúa en el ámbito local a través de los cursos de especialización disciplinar (Intervención Comunitaria I y II) para profundizar en las preocupaciones locales, que se centran exclusivamente en el tema de la migración:

(...) los estudiantes, acompañados por un profesor guía, realizarán un diseño e implementarán un programa de intervención. Este proceso incluirá: el diagnóstico y la contextualización, formulación de objetivos, la determinación colaborativa de los componentes y contenidos del programa de intervención, su fundamentación teórica, la selección de los participantes del programa y de las estrategias de intervención, la ejecución de estas estrategias, los métodos para evaluar el logro de objetivos, el procesamiento de la información y análisis de datos, evaluación, seguimiento y la sustentabilidad del proyecto. (Programa curso Intervención Comunitaria I, 2011)

Investigación e innovación. A través de sus docentes, en el área se implementa una línea de investigación en migración que entrega insumos a las actuales y futuras intervenciones, que se desarrollan en el marco de acciones de vinculación con el medio, por un lado, y a la Oficina de Migración, por otro. La Oficina de Migración se crea el año 2015 y corresponde a la institucionalidad local encargada de desarrollar acciones que apoyen a la población migrante residente en la comuna, además de diseñar e implementar la política local de migración (antes de la creación de esta oficina, el gobierno local, canalizaba las acciones en esta materia a través del Departamento de Organizaciones Comunitarias). Junto con ello, ejecuta una línea de trabajo en torno a la convivencia vecinal, que pretende resolver las dificultades cotidianas que experimentan los barrios cuando interactúa la población nacional con la extranjera.

Estas investigaciones han sido desarrolladas a través de las asignaturas Proyecto y Seminario de Título, y a través de los fondos de financiamiento interno que se postulan anualmente al interior de la universidad.

Es importante destacar que, para todas las investigaciones implementadas, se trabajó la dimensión ética y política puesta en juego en cualquier proceso investigativo donde participen sujetos en condición de minoría, como es el caso de los inmigrantes, niños, mujeres u homo/transexuales, aspecto que resulta consistente con la perspectiva valórica que aporta la PC.

En la Tabla 2 se resumen las investigaciones desarrolladas hasta la fecha.

Tabla 2

Resumen de Investigaciones Desarrolladas en el Área

Nombre investigación

Asignaturas de malla curricular: Proyecto y Seminario de Investigación

La plaza el trébol: tú, yo y nosotros. Prácticas interculturales de niños y niñas en un espacio público de Independencia, un estudio de caso en Chile

Migración peruana, cuidado e interculturalidad: la influencia de inmigrantes peruanas trabajadoras de casa particular en familias chilenas

Relaciones familiares y bienestar subjetivo en el campo biográfico: un acercamiento a la experiencia migratoria de un grupo de personas peruanas que residen en Chile alejadas de sus grupos familiares de origen y procreación

Migrantes del Perú: servicios de cuidado doméstico, bajo la mirada de la justicia cultural

Hacer y sistematizar: una reflexión sobre el proceso vivido en la intervención social realizada en la Comuna de Independencia

Cadenas de cuidado transnacionales ¿Cómo cuidamos a quien nos cuida?

Doble discriminación: perspectiva de migrantes latinoamericanos pertenecientes a diversidades sexuales en Chile

Valoración y percepción de estudiantes chilenos hacia migrantes peruanos, respecto a la entrega de conocimientos, cultura y experiencias que enriquecen al país de destino

Concurso interno

Impacto de los procesos migratorios peruanos hacia Chile en la configuración de las cadenas globales de cuidado: un estudio exploratorio-descriptivo en la comuna de Independencia
20142015

$2014-$

2015

2014-

2015

2014-

2015

2014-

2015

2015-

2016

2015-

2016

$2015-$

2016

2014- Equipo investigadoras,

2015 Escuela de Psicología

Estudiantes Psicología

Estudiantes Psicología

Estudiantes Psicología

Estudiantes Psicología

Estudiantes Psicología

Estudiantes Psicología

Estudiantes Psicología

Estudiantes Psicología 
Cabe destacar que los resultados de estas investigaciones se hicieron llegar a los actores locales a través de una instancia de intercambio, en la que participaron los miembros de la Oficina de Migración, representantes del departamento de educación municipal, estudiantes de los cursos de Intervención Comunitaria I y II y miembros de organizaciones de migrantes convocados especialmente para estos efectos. La intención fue que el conocimiento producido en este ámbito sirviera como un insumo relevante para la generación de acciones comunitarias y para promover una reflexión colectiva sobre la inmigración y sus implicancias en el quehacer local.

\section{Propuesta Metodológica}

Se describen las acciones comunitarias que se han llevado a cabo a lo largo del proceso formativo, junto a las prácticas docentes que imprimen un cierto sello a la formación recibida por los estudiantes y, finalmente, se da cuenta de los resultados alcanzados que permiten comprender para qué sirve la propuesta implementada desde el área.

Acciones implementadas. Las principales acciones que se llevan a cabo para concretar esta propuesta formativa se orientan a instalar una relación de cooperación con el departamento de Organizaciones Comunitarias de la Municipalidad de Independencia, que funcionó como contraparte hasta que se formalizó la Oficina de Migración como orgánica local. Esta primera acción fue relevante, debido a que se llevó a cabo el primer catastro de viviendas, que hizo posible que estudiantes del curso de Diagnóstico Social Comunitario participaran del diseño, implementación y análisis de una encuesta de habitabilidad que permitió al municipio acceder a datos de caracterización, aún no disponibles debido a las dificultades que tuvo el proceso censal llevado a cabo por el Instituto Nacional de Estadísticas el año 2012 (El XVIII Censo Nacional de Población y VII de Vivienda o Censo de Población y Vivienda 2012 fue realizado por el INE, aunque su uso debió ser desestimado oficialmente en 2014, debido a una serie de problemas en la elaboración y tratamiento de los datos. De acuerdo a la auditoría interna liderada por el INE y el Centro Latinoamericano de Demografía, los datos generados el año 2012 no cumplían con los estándares para que este operativo fuera considerado un censo). Asimismo, y dentro de este proceso de encuesta, el equipo docente decide aplicar un instrumento paralelo, que buscaba mapear los recursos comunitarios existentes en el territorio. Estos datos funcionaron como insumo para el período posterior, en el que los estudiantes de los cursos de Evaluación de Proyectos Sociales propusieron proyectos de intervención que luego evaluaron participativamente con la comunidad. Finalmente, los estudiantes que deciden ser parte del área social comunitaria emprenden una relación directa con la recientemente creada Oficina de Migración de Independencia, dando continuidad a los procesos de intervención territorial ya desarrollados en etapas previas. En la Tabla 3 se sintetizan las principales acciones con sus respectivas metodologías.

Respecto de las acciones implementadas en el ámbito de la migración, los interventores señalan que se trataba de un desafío relevante para ellos, en tanto cambia el contexto de trabajo y deben implementar nuevas estrategias para las que no están preparados. Dichas estrategias buscan caracterizar los vecindarios multiculturales y conocer los modos en que la población nacional interactúa con la población migrante, detectando a través de diagnósticos, necesidades y recursos.

Las demandas de la comunidad que cada vez son más diversas, pues en esta comuna ya prácticamente el $50 \%$ de la población es de origen migrante. (E 8, p. $1^{2}$ )

Vino esta llegada masiva de los nuevos vecinos (...) [nosotros nos encontrábamos] con poco conocimiento de cómo intervenir (...) sin saber qué podíamos hacer. (E 7, p. 2)

Como yo, por ejemplo, las personas que estábamos encargadas no teníamos mayor conocimiento de migrantes. (E 7, p. 2)

\footnotetext{
2 Para efectos del reporte de resultados, a cada entrevista se le asignó un número (del 1 al 7). Las citas textuales incluyen esta información (por ejemplo, E 1 = Entrevista 1), seguido por el número de página de la cita.
} 
Tabla 3

Acciones Comunitarias Llevadas a Cabo en el Marco del Proceso Formativo

\begin{tabular}{|c|c|c|c|}
\hline Asignatura & Semestre & Actividad & Metodologías y acciones \\
\hline $\begin{array}{l}\text { Diagnóstico } \\
\text { Social } \\
\text { Comunitario }\end{array}$ & $\begin{array}{l}2014 \text { y } 2015 \\
\text { II semestre }\end{array}$ & $\begin{array}{l}\text { Catastro habitabilidad y mapa de recursos. } \\
\text { Diagnóstico de situación de vecinos migrantes } \\
\text { y relación con Oficina Municipal de Migración. }\end{array}$ & $\begin{array}{l}\text { Familiarización, diseño y aplicación de } \\
\text { técnicas cualitativas, entrevistas, grupos } \\
\text { focales, mapeo, análisis de datos. } \\
\text { Sistematización de datos para el diseño de } \\
\text { intervenciones comunitarias. }\end{array}$ \\
\hline $\begin{array}{l}\text { Evaluación de } \\
\text { Proyectos } \\
\text { Sociales }\end{array}$ & $\begin{array}{l}2015 \text { y } 2016 \\
\text { I semestre }\end{array}$ & $\begin{array}{l}\text { Intervenciones a partir del diagnóstico en } \\
\text { temas como convivencia vecinal, difusión de la } \\
\text { Oficina de Migración, preparación para el } \\
\text { trabajo, información sobre regularización de } \\
\text { situación migratoria, derechos de migrantes, } \\
\text { entre otros. }\end{array}$ & $\begin{array}{l}\text { Negociación de la demanda para acordar, con } \\
\text { la participación de todos los actores, la } \\
\text { intervención, se desarrollan talleres, } \\
\text { capacitaciones, reuniones ampliadas, } \\
\text { asambleas, uso de plazas y sedes vecinales, así } \\
\text { como casas de migrantes. }\end{array}$ \\
\hline $\begin{array}{l}\text { Intervención } \\
\text { Comunitaria I }\end{array}$ & $\begin{array}{l}2014 \text { y } 2015 \\
\text { II semestre }\end{array}$ & $\begin{array}{l}\text { Desarrollo de proyectos participativos en } \\
\text { polígono territorial definido por municipio: } \\
\text { temas recuperación de espacio público, } \\
\text { participación comunitaria, convivencia } \\
\text { vecinal, conocer al vecino. }\end{array}$ & $\begin{array}{l}\text { Acercamientos progresivos con organizaciones } \\
\text { de migrantes y nacionales para convocar a } \\
\text { mesas de trabajo. Acciones complementarias a } \\
\text { la estrategia municipal para facilitar la } \\
\text { integración de migrantes y nacionales. }\end{array}$ \\
\hline $\begin{array}{l}\text { Intervención } \\
\text { Comunitaria II }\end{array}$ & $\begin{array}{l}2015 \text { y } 2016 \\
\text { I semestre }\end{array}$ & $\begin{array}{l}\text { Cierre de procesos de intervención } \\
\text { correspondientes a los iniciados en diagnóstico } \\
\text { social comunitario. } \\
\text { Promoción de la participación de nacionales y } \\
\text { extranjeros. } \\
\text { Promoción y entrega de información: ser } \\
\text { migrante en Chile. }\end{array}$ & $\begin{array}{l}\text { Talleres huertos comunitarios, convocatoria a } \\
\text { mesa de trabajo territorial como estrategia } \\
\text { complementaria al municipio, murales, fiestas } \\
\text { y pasacalles. } \\
\text { Información sobre situación legal en } \\
\text { colaboración con la carrera de derecho. }\end{array}$ \\
\hline
\end{tabular}

Nota. Desde el año 2014 hasta la fecha, el promedio anual de personas que han participado en los distintos ámbitos (territorio, municipio, universidad) es de 180 personas, distribuidos en las diferentes asignaturas, con alrededor de 16 proyectos o intervenciones por semestre, con aproximadamente 90 vecinos, 100 estudiantes, cinco docentes y tres profesionales municipales.

Prácticas docentes. En la propuesta que ahora se presenta, la labor de los docentes ha sido fundamental, pues permite implementar un modo de relación que invita a los estudiantes a repensar su lugar en la formación universitaria. Al mismo tiempo, se trata de docentes que cuentan con un compromiso por la labor realizada, acompañando actividades en terreno y poniendo a disposición de los estudiantes un dispositivo formativo de supervisión que integra distintas dimensiones al proceso (desarrollo personal y profesional, mejoramiento de capacidad técnica, reflexión ética, entre otras).

Uno de los docentes del área comunitaria señala lo siguiente:

Uno de los factores más relevantes que facilita el cumplimiento de objetivos es la modalidad de la clase, pues permite un acompañamiento permanente y personalizado al trabajo que realiza en terreno cada dupla de trabajo. Allí se juegan habilidades, como la autonomía y el trabajo en equipo. (Docente, Intervención Comunitaria I, 2016)

Otro docente agrega que:

(...) también se presentan resistencias que impiden el desempeño óptimo, pues se produce una ruptura con el formato tradicional de la formación universitaria que tiende a ubicar al estudiante en un lugar pasivo, como un mero receptor de conocimientos (...) En esta modalidad de trabajo, se interpela a los estudiantes a que sean ellos los productores del conocimiento y, por tanto, se vuelven protagonistas de su proceso formativo. (Docente, Intervención Comunitaria I, 2016)

A su vez, los propios estudiantes destacan de su formación las capacidades y el trabajo de los docentes. Acá se incluye el testimonio de los estudiantes provenientes de la primera generación que participó del plan curricular que ahora se analiza:

(...) es que la formación que recibí es más de los docentes que de la institución o de las mallas, porque por lo general de ahí se desprende la experiencia que no estamos teniendo nosotros mismos como protagonistas. (Grupo Focal, estudiantes, 2014)

Al mismo tiempo, los estudiantes manifiestan que se vuelve relevante que los docentes logren poner en valor la praxis como una dimensión fundamental del quehacer en PC:

(...) entonces, siento que como que pasó eso, que la psicología comunitaria no separa la teoría y la práctica, sino que iban a la par y, a medida que íbamos viendo algo acá en terreno, también íbamos viendo algo en lo teórico, 
entonces, eso facilita mucho más el aprendizaje y lo significa mucho más, o sea, no es una cosa que uno aprenda en una prueba y después se le olvida, sino que queda en el inconsciente lo que hay que hacer. (Grupo Focal, estudiantes, 2014)

Valoración de las acciones implementadas. Destaca como relevante la posibilidad de incidir a nivel territorial, para colaborar en la generación de una política local que surge desde la consideración a las necesidades de la comunidad. En este sentido, los cursos de Intervención Comunitaria I y II se han dedicado a colaborar en las acciones locales emprendidas desde la Oficina de Migración en un plazo de tres años, logrando fortalecer el trabajo territorial que se desarrolla en la comuna.

Los propios funcionarios locales que fueron parte del desarrollo de las intervenciones destacan que:

[el trabajo de los estudiantes] fue súper importante para llevar un trabajo que a nosotros nos había costado bastante. (E 6, p. 1)

[los estudiantes] están dispuestos a hacer un trabajo comunitario que nos sirve para después ir avanzando en nuestras funciones. (E 7, p. 1)

Al mismo tiempo, para los propios participantes de las intervenciones se trató de una experiencia significativa, en tanto permitió un intercambio de saberes y conocimientos:

Aprendí mucho de ellos y además incorporaron tantas cosas positivas, que te juro que yo echo mucho de menos.

(E 5, p. 1)

Excelente, fue muy bueno, la gente muy cooperadora, cuando había que pedirle algún favor para que lo ayudaran, ellos siempre estuvieron dispuestos. (E 1, p. 1)

Limitación de la experiencia. Los actores participantes del proceso destacan, a modo de evaluación, algunos aspectos negativos que constituyen una retroalimentación interesante para el mejoramiento de los programas y currículos, en cuanto a límites de tiempo y alcance del trabajo propuesto.

Algunos participantes entrevistados señalan:

Yo le diría a la universidad que no saque a los alumnos cuando ya estamos comprometidos, porque dejan la tarea a medias. (E 1, p. 1)

Nunca más nadie hizo nada [con los niños y niñas participantes de la intervención] y los chiquillos preguntaban qué pasaba y todo eso, entonces, quedamos como en el aire. (E 5, p. 1)

Por su parte, los funcionarios públicos también agregan una visión crítica, que profundiza lo planteado por los participantes:

Pero que la universidad y los profesionales sigan interviniendo, o sea, no dejen el proceso botado. (E 6, p. 2)

Entonces no se puede hacer como una intervención más profunda o planificada, porque los profesores tienen su propia planificación. (E 7, p. 1)

\section{Componentes de la Formación en PC}

Desde el año 2014, el equipo docente del área social comunitaria decide emprender el desafío de formar a estudiantes sensibles al problema de la migración, en el que la intervención comunitaria funciona como estrategia de abordaje preferencial.

Propuesta curricular. En este aspecto se define que las acciones llevadas a cabo en el territorio sigan el criterio de continuidad temporal para establecer un trabajo que pueda ser supervisado y evaluado en términos de su impacto. Dentro de la malla curricular, en el segundo semestre de 2014 se asigna a la asignatura de Diagnóstico Social Comunitario la tarea de levantar información y datos de la comuna de Independencia en torno a la migración. De forma paralela, el curso de Intervención Social Comunitaria I comienza los primeros acercamientos a territorios propuestos por el municipio para iniciar un trabajo, por lo que se les asigna un polígono determinado (delimitación territorial definida por el municipio de Independencia) que cuenta con una presencia de alta población migrante, teniendo como objetivo central recoger información para iniciar procesos de intervención.

Luego, en el primer semestre del año 2015, la asignatura de Evaluación de Proyectos Sociales da continuidad a lo realizado en Diagnóstico Social Comunitario, tomando los datos recogidos por este curso, para proponer intervenciones que se orienten a responder lo planteado por la comunidad. Asimismo, Intervención Social Comunitaria II continúa con el proceso de su antecesor, desarrollando acciones en el 
espacio territorial ya definido. Por lo tanto, es posible realizar un trabajo que tiene como mínimo de duración un año, pudiendo llegar hasta tres años, situando de manera paralela las asignaturas de Proyecto y Seminario de Investigación, que, como ya se describió en la Tabla 4, tiene por objetivo situar la temática migración como un foco necesario de investigar desde la PC. Para llegar al tercer año de trabajo conjunto en el mismo territorio, se requirió la instalación de la Práctica Profesional del área social comunitaria, lo que implicó tener a un estudiante que había sido parte del proceso desde Diagnóstico Social Comunitario, por lo tanto, tenía en su formación elementos que daban una base para llevar a cabo su práctica profesional en la Oficina de Migración de Independencia. Ver el detalle en la Tabla 4.

Tabla 4

Acciones Territoriales y Comunitarias Desarrolladas a Partir de la Propuesta Pedagógica

\begin{tabular}{|c|c|c|}
\hline Nombre del curso & Objetivo & Etapa \\
\hline Diagnóstico Social Comunitario & $\begin{array}{l}\text { Levantar información y datos de la comuna de Independencia en torno a la } \\
\text { migración. }\end{array}$ & $2014 / 2^{\circ}$ sem. \\
\hline Intervención Social Comunitaria I & $\begin{array}{l}\text { Recoger información para iniciar procesos de intervención y darle } \\
\text { continuidad a la intervención. }\end{array}$ & $2014 / 2^{\circ}$ sem. \\
\hline Evaluación de Proyectos Sociales & $\begin{array}{l}\text { Tomando los datos recogidos por el curso Diagnóstico Social Comunitario, } \\
\text { proponer intervenciones que se orienten a responder a las necesidades } \\
\text { detectadas con los recursos de la comunidad y los profesionales. }\end{array}$ & $2015 / 1^{\circ}$ sem. \\
\hline Intervención Social Comunitaria II & $\begin{array}{l}\text { Continuidad mediante el desarrollo de acciones en el espacio territorial ya } \\
\text { definido. }\end{array}$ & $2015 / 1^{\circ}$ sem. \\
\hline Proyecto de Investigación & $\begin{array}{l}\text { Construir un problema de investigación abordable desde la psicología que } \\
\text { permita comprender el fenómeno de la migración expresado en el ámbito local. }\end{array}$ & $2015 / 2^{\circ}$ sem. \\
\hline Intervención Social Comunitaria I & $\begin{array}{l}\text { Colaboración técnica para implementar estrategia local desde la Oficina de } \\
\text { Migración de Independencia. }\end{array}$ & $2015 / 2^{\circ}$ sem. \\
\hline Seminario de Investigación & $\begin{array}{l}\text { Implementar el diseño propuesto en la etapa anterior, a través de una } \\
\text { metodología preferentemente cualitativa, que busque producir información } \\
\text { sobre el fenómeno migratorio en el ámbito local. }\end{array}$ & $2016 / 1^{\circ}$ sem. \\
\hline Intervención Social Comunitaria II & $\begin{array}{l}\text { Desarrollo de acciones comunitarias locales con vecinos, dirigentes y } \\
\text { organizaciones. }\end{array}$ & $2016 / 1^{\circ}$ sem. \\
\hline Práctica Profesional & $\begin{array}{l}\text { Insertarse en el gobierno local (Oficina de Migración) para colaborar en la } \\
\text { generación de condiciones para la producción de una política local en migración. }\end{array}$ & $\begin{array}{l}2016 / 2^{\circ} \text { sem. } \\
2015 / 2^{\circ} \text { sem. }\end{array}$ \\
\hline
\end{tabular}

Principios y valores de la propuesta. En primer lugar, esta propuesta incluye, como condición, una alianza de colaboración entre el mundo privado y público, reflejado en la universidad como institución privada y el Municipio de Independencia, como entidad pública local. Se define como una alianza basada en la cooperación, en la que la universidad presta servicios a la comunidad y el municipio asegura el trabajo en sus territorios para responder a los temas de interés y preocupación local y nacional.

Por otro lado, desde lo académico, uno de los criterios básicos de las intervenciones desde el área social comunitaria se relaciona con la realización de acciones en las que la participación de todos los actores posibles sea una constante (vecinos nacionales, extranjeros, niños, jóvenes, dirigentes, organizaciones sociales, profesionales, entre otros), pudiendo acceder a todos los puntos de vista que construyen la realidad dada, sin privilegiar a uno en desmedro de otro.

De este modo, una de las asignaturas que forma parte del ciclo de especialización señala como elementos actitudinales a entrenar:

Respeto y ética en las relaciones y comunicaciones con las personas involucradas en la intervención; Integridad, honestidad y legitimidad en el proceso de intervención, incluyendo elecciones de técnicas interventivas que no afecten valores comunitarios; Atención a posibles efectos secundarios a terceros que no han solicitado ayuda ni intervenido en decisiones del programa. (Programa curso Intervención Comunitaria I, 2011)

En el mismo texto se destina una unidad completa a tratar este ámbito:

Unidad IV: Dimensión ética y política de las intervenciones socio-comunitarias, [siendo resultados de aprendizaje]: Analizar las tensiones de la práctica comunitaria en el escenario nacional y mundial actual, [junto con] Analizar la acción comunitaria orientada al cambio social y la coherencia entre teoría y práctica. (Programa curso Intervención Comunitaria I, 2011) 
Por su parte, los propios estudiantes destacan que existen valores fundamentales en la formación en PC recibida:

(...) la horizontalidad... yo igual siento que es un tema importante, sobre todo porque nos sacamos... no sé si nos sacamos por completo el sombrero y el traje, pero nos sacamos la capa, y eso ya implica trabajar de una manera distinta con este contexto de intervención, o sea, se reduce la posición de poder en la cual de alguna manera nos forman. (Grupo Focal, estudiantes, 2014)

(...) personalmente y a nivel político encuentro que lo estandarizado no sirve, que no hay una realidad objetiva, porque todos vemos diferentes tonos en los colores, entonces, la contextualización nos ayuda a saber con qué color ven las personas con las que trabajo y no trabajar desde nuestro azul carmesí. (Grupo Focal, estudiantes, 2014)

\section{Discusión y Conclusiones}

Se considera que la PC es una subdisciplina que se valida, en primer lugar, por constituir un tipo de práctica crítica que interpela de manera transversal a la psicología, tanto en su dimensión teórica como en su quehacer práctico (Rodríguez Kauth, 2008). Es a partir de esta interpelación, que se propone revisar las prácticas formativas (Farías, 2009) e innovar en una propuesta que articule teoría y práctica y que rescate las fortalezas existentes en las disposiciones institucionales donde la relación universidad-comunidad (Montero \& Serrano-García, 2011) se vuelve deseable y necesaria para producir cambios y mejoras en los planes y políticas locales.

Es en este contexto donde se fortalece una práctica docente que admite la horizontalidad en la relación con los estudiantes, invitando a que estos asuman un rol activo y participativo en su proceso formativo (Montero \& Giuliani, 1997; Riveros, 2012), posición que, en general, no es estimulada en la educación chilena actual.

Del mismo modo, otro ámbito que se ve fuertemente impactado, es el de los propios pares que, como docentes, se espera que propongan, cuestionen, exploren y se apropien de su tarea formativa, en una lógica colaborativa y de cooperación. Al mismo tiempo, también se demanda un alto nivel de compromiso y de participación activa en el proceso, supervisando y revisando constantemente las intervenciones realizadas por los estudiantes.

Por otro lado, y como parte del contexto de formación en PC existente en Chile, se coincide con lo propuesto por distintos autores (Winkler et al., 2015) al momento de constatar que las lógicas de intervención tienden a situar el rol profesional en términos de experto, legitimando el saber profesional y académico como el único autorizado para comprender e intervenir en la realidad, desconociendo la existencia y legitimidad de otros saberes, como el popular, ampliamente valorado en los orígenes de la PC, como una fuente de conocimiento irremplazable. Esto se vuelve aún más crítico, si se considera que actualmente y como efecto de la aplicación de políticas asistenciales provenientes de la actuación del estado neoliberal vigente (Reyes Espejo et al., 2015), las comunidades tienden a demandar soluciones rápidas y efectivas, sin implicarse como actores que participan también de la solución a sus problemas y exigencia de derechos. Entonces, por un lado, el profesional no estimula la emergencia de un saber comunitario y, por otro, los miembros de las comunidades no demandan participar como actores y se dejan intervenir de manera pasiva, acrítica e instantánea (Prilleltensky, 2001).

Respecto al contexto en el que se desarrolla la propuesta formativa, resulta interesante destacar la flexibilidad de los currículos institucionales que permitieron incluir el análisis de la realidad social y, en particular, de la migración, como un escenario en el que se despliegan aprendizajes situados y se preparan contenidos pertinentes a este contexto (Yañez Canal, 2005). Del mismo modo, se trata de un proyecto formativo que colabora en la generación de respuestas urgentes ante un problema complejo y que incluye a un cada vez más amplio sector de la sociedad, generando un malestar que no ha sido abordado y que desencadena efectos en múltiples niveles, escasamente atendidos hasta el momento (Tijoux \& Palominos Mandiola, 2015).

Considerando lo anterior, se vuelve necesario fortalecer y consolidar la colaboración técnica ofrecida desde el área social comunitaria para el ámbito de la migración, potenciando la producción de conocimiento situado y pertinente a los contextos, que pueda dialogar de manera más directa con los procesos de intervención que se puedan implementar. En este sentido, es importante reconocer que la vinculación con lo público es valorada desde el marco universitario; no obstante, requiere de una alta inversión de tiempo y de recursos humanos y económicos, por lo tanto, de actores que se vinculen de manera sistemática destinando tiempo y estableciendo compromisos de mediano y largo plazo (Rodríguez, Pérez, Prieto, \& López, 2015), tal 
como lo propone el marco dado por la metodología $\mathrm{A}+\mathrm{S}$, que aún no se termina de formalizar completamente en Chile.

Del mismo modo, los distintos actores reconocen aprendizajes significativos en cuanto a madurar la comprensión del fenómeno migratorio, adecuando abordajes que en un principio solo incorporaban un trabajo focalizado con la población migrante (fundamentalmente, de nacionalidad peruana, colombiana y haitiana), para luego ampliar el foco e incluir a los nacionales, abordando de manera relacional y compleja el problema. Este proceso fue posible debido a la permanencia en el territorio, que aseguró la continuidad de la intervención por al menos tres años. Cabe destacar que la permanencia, deseable desde el ámbito académico, no siempre se encuentra garantizada, debido a los tiempos políticos que marcan el espacio local, producto de los procesos eleccionarios que interfieren directamente en las intervenciones comunitarias en curso (Winkler, Alvear, Olivares \& Pasmanik, 2009). Considerando que los propios actores y funcionarios entrevistados también visualizan negativamente la discontinuidad temporal de las intervenciones, este resulta un aspecto particularmente crítico y sensible a la hora de pensar en procesos de formación en el campo, desde una perspectiva ético-política (Winkler et al., 2014).

Esta propuesta invita a pensar en el rol social de la universidad (Rodríguez et al., 2015) y, con ello, en las condiciones de producción de conocimiento, que determinan para quién se trabaja, desde dónde y cómo se considera al otro en esta relación de servicio y colaboración. Así, se concreta una conversación en la que todos los actores sociales se convocan, acercando la academia a los contextos comunitarios e institucionales, fortaleciendo una reflexión ético-política necesaria para consolidar una propuesta que recupere los valores fundantes de la disciplina (Prilleltensky, 2001; Montero, 2010a, 2010b).

Una tarea pendiente es evaluar y analizar críticamente el impacto en la formación de estos estudiantes y el efecto en la construcción de su rol y ethos profesional y, por ende, en sus prácticas socio-profesionales futuras. Del mismo modo, es necesario continuar los procesos de intervención, buscando concretar cambios y generar consecuencias transformadoras en las comunidades que han sido parte del proceso. Se asume, con todo, el desafío permanente de repensar los dispositivos de formación (Rodríguez et al., 2015) para estimular y desarrollar una psicología al servicio de las necesidades y recursos de las comunidades, comprometida con la transformación más que con el mejoramiento social (Reyes Espejo et al., 2015), que pueda hacerse cargo de dar respuesta a los problemas más sentidos, respetando singularidades y proponiendo modos de abordaje integrales y complejos.

\section{Referencias}

Asún, M. (2005). Para leer la psicología comunitaria: contextualización histórica en Santiago de Chile (Tesis de Grado no publicada), Facultad de Psicología, Universidad Diego Portales, Santiago, Chile.

Banyard, V. L. \& Miller, K. E. (1998). The powerful potential of qualitative research for community psychology. American Journal of Community Psychology, 26, 485-505. https://doi.org/10.1023/A:1022136821013

Bilbeny, N. (2004). Ética intercultural: la razón práctica frente a los retos de la diversidad cultural. Barcelona, España: Ariel.

Bolívar, A. (2005). El lugar de la ética profesional en la formación universitaria. Revista Mexicana de Investigación Educativa, 10(24), 93-123. Extraído de http://www.scielo.org.mx/scielo.php?script=sci_arttext\&pid=S1405-66662005000100093

Canales Cerón, M. (Ed.) (2006). Metodologías de investigación social: introducción a los oficios. Santiago, Chile: LOM.

Casanova, F. (2003). Formación profesional y relaciones laborales. Montevideo, Uruguay: Cinterfor/Organización Internacional del Trabajo.

Castillo, J. \& Winkler, M. I. (2010). Praxis y ética en psicología comunitaria: representaciones sociales de usuarias y usuarios de programas comunitarios en la Región Metropolitana. Psykhe, 19(1), 31-46. https://doi.org/10.4067/s0718-22282010000100003

Comisión Nacional de Acreditación-Chile (2013). Aprueba reglamento sobre áreas de acreditación acorde al artículo $17^{\circ}$ de la Ley 20.129. Santiago, Chile: Autor. Extraído de https://www.cnachile.cl/Documentos\%20de\%20Paginas/RES-DJ-01.pdf

Cortina, A. (2001). Ética aplicada y democracia radical. Madrid, España: Tecnos.

Dulzaides Iglesias, M. E. \& Molina Gómez, A. M. (2004). Análisis documental y de información: dos componentes de un mismo proceso. Acimed, 12(2), Contribuciones Breves 3. Extraído de http://scielo.sld.cu/scielo.php?script=sci_arttext\&pid=S1024-94

Farías, F. (2009). La epistemología de las ciencias sociales en la formación por competencias del pregrado. Cinta de Moebio, 34, 58-66. https://doi.org/10.4067/s0717-554x2009000100004

Instituto Nacional de Estadísticas-Departamento de Extranjería y Migración (2019). Estimación de personas extranjeras residentes en Chile al 31 de diciembre 2018. Informe Metodológico. Extraído de https://www.extranjeria.gob.cl/media/2019/07/Estimaci\%C3\%B3nPoblaci\%C3\%B3n-Extranjera-en-Chile.pdf

Íñiguez Rueda, L. (1999). Investigación y evaluación cualitativa: bases teóricas y conceptuales. Atención Primaria, 23, 496- 502. Extraído de https://www.elsevier.es/es-revista-atencion-primaria-27-articulo-investigacion-evaluacion-cualitativa-bases-teoricas-14823

Krause, M., Jaramillo Torrens, A., Monreal, V., Carvacho García, H. \& Torres, A. (2011). Historia de la psicología comunitaria en Chile: desde la clandestinidad a la política pública. En M. Montero \& I. Serrano-García (Comps.), Historias de la psicología comunitaria en América Latina: participación y transformación (pp. 115-138). Buenos Aires, Argentina: Paidós.

Martínez, M., Buxarrais, M. R. \& Bara, F. E. (2002). La universidad como espacio de aprendizaje ético. Revista Iberoamericana de Educación, 29, 17-43. https://doi.org/10.35362/rie290949 
Martínez Carazo, P. C. (2006). El método de estudio de caso: estrategia metodológica de la investigación científica. Pensamiento \& Gestión, 20, 165-193. Extraído de https://www.redalyc.org/pdf/646/64602005.pdf

Martinic, S. (2010). El objeto de la sistematización y sus relaciones con la evaluación y la investigación. Medellín, Colombia: Fundación Universitaria Luis Amigó/Consejo de Educación Popular de América Latina y el Caribe. Extraído de http://centroderecursos.alboan.org/ebooks/0000/0748/6_CEA_OBJ.pdf

Mieles Barrera, M. D., Tonon, G. \& Alvarado Salgado, S. V. (2012). Investigación cualitativa: el análisis temático para el tratamiento de la información desde el enfoque de la fenomenología social. Universitas humanística, 74, 95-225. Extraído de http://www.scielo.org.co/pdf/unih/n74/n74a10.pdf

Montero, M. (2006). Hacer para transformar: el método en la psicología comunitaria. Buenos Aires, Argentina: Paidós.

Montero, M. (2010a). Unión de actores sociales, participación comunitaria y ética, en la ejecución de políticas públicas. En A. E. Hincapié Gómez (Comp.), Sujetos políticos y acción comunitaria: claves para una praxis de la psicología social y de la clínica social comunitaria en América Latina (pp. 45-66). Medellín, Colombia: Universidad Pontificia Bolivariana.

Montero, M. (2010b). Fortalecimiento de la ciudadanía y transformación social: área de encuentro entre la psicología política y la psicología comunitaria. Psykhe, 19(2), 51-63. https://doi.org/10.4067/s0718-22282010000200006

Montero, M. \& Giuliani, F. (1999). La docencia en la psicología social comunitaria: algunos problemas. Psykhe, 8(1), 57-63. Extraído de http://www.psykhe.cl/index.php/psykhe/article/view/396/376

Montero, M. \& Serrano-García, I. (Comps.) (2011). Historias de la psicología comunitaria en América Latina. Participación y Transformación. Buenos Aires, Argentina: Paidós.

Olivares, B., Reyes, M. I., Berroeta, H. \& Winkler, M. I. (2016). La formación universitaria en la psicología comunitaria chilena de hoy: ¿un lugar subalterno? Psykhe, 25(2), 1-12. https://doi.org/10.7764/psykhe.25.2.868

Prilleltensky, I. (2001). Value-based praxis in community psychology: Moving toward social justice and social action. American Journal of Community Psychology, 29, 747-778. https://doi.org/10.1023/a:1010417201918

Reyes Espejo, M. I., Olivares Espinoza, B., Berroeta Torres, H. \& Winkler Müller, M. I. (2015). Del discurso a las prácticas: políticas sociales y psicología comunitaria en Chile. Polis, 42, 1-22. https://doi.org/10.4067/s0718-65682015000300018

Riveros, P. (2012). Formación profesional de pregrado en psicología comunitaria en Santiago de Chile en la actualidad: análisis descriptivo de los contenidos de enseñanza, metodologías y perfil profesional del psicólogo/a comunitario (Tesis de Magíster no publicada), Facultad de Ciencias Sociales, Universidad de Chile, Santiago, Chile.

Rodríguez, A., Pérez, L., Prieto, M. \& López, S. (2015). Caminos en la formación en psicología social comunitaria: procesos y rupturas. Psicología, Conocimiento y Sociedad, 5(2), 259-277. Extraído de http://www.scielo.edu.uy/scielo.php?script=sci_arttext\&pid=S1688-70262015000100011

Rodríguez Kauth, A. (2008). La investigación y enseñanza en psicología política. Revista Electrónica de Psicología Política, 6(17), 1-11. Extraído de http://www.psicopol.unsl.edu.ar/julio2008_nota6.pdf

Rozas, G. \& Martínez, V. (2007). Sobre el nacimiento, desarrollo y proyecciones de la psicología comunitaria en la Universidad de Chile. En A. Zambrano Constanzo, G. Rozas Ossandón, I. Magaña Frade, D. Asún Salazar \& R. Pérez-Luco Arenas (Eds.), Psicología comunitaria en Chile: evolución, perspectivas y proyecciones (pp. 57-76). Santiago, Chile: RIL.

Sassen, S. (2000/2003). Contrageografías de la globalización: género y ciudadanía en los circuitos transfronterizos. (A. Pastrana, Trad.; Título original: Women's Burden: Counter-geographies of Globalization and the Feminization of Survival). Madrid, España: Traficantes de Sueños.

Stein, C. H. \& Mankowski, E. S. (2004). Asking, witnessing, interpreting, knowing: Conducting qualitative research in community psychology. American Journal of Community Psychology, 33, 21- 35. https://doi.org/10.1023/B:AJCP.0000014316.27091.e8

Tapia, M. N. (2006). Aprendizaje y servicio solidario. En las instituciones educativas y las organizaciones juveniles. Buenos Aires, Argentina: Ciudad Nueva.

Tijoux, M. E. \& Palominos Mandiola, S. (2015). Aproximaciones teóricas para el estudio de procesos de racialización y sexualización en los fenómenos migratorios de Chile. Polis, 14(42), 1-23. https://doi.org/10.4067/S0718-65682015000300012.

Vasquéz de Kartzow, R. (2009). Impacto de las migraciones en Chile. Nuevos retos para el pediatra. ¿Estamos preparados? Revista Chilena de Pediatria, 80(2), 161-167. Extraído de http://repositorio.uchile.cl/bitstream/handle/2250/129047/Impacto-de-lasmigraciones-en-Chile.pdf?sequence $=1$

Vietyes, R. (2009). Campos de aplicación y decisiones de diseño en la investigación cualitativa. En A. Merlino (Coord.), Investigación cualitativa en ciencias sociales: temas, problemas y aplicaciones (pp. 41-84). Buenos Aires, Argentina: Cengage Learning.

Winkler, M. I., Alvear, K., Olivares, B. \& Pasmanik, D. (2009). Autonomía versus dependencia. Quehacer comunitario y politicas públicas. Santiago, Chile: Universidad de Santiago de Chile, Vicerrectoría de Investigación.

Winkler, M. I., Alvear, K., Olivares, B. \& Pasmanik, D. (2014). Psicología comunitaria hoy: orientaciones éticas para la acción. Psicoperspectivas, 13(2), 44-54. https://doi.org/10.5027/PSICOPERSPECTIVAS-VOL13-ISSUE2-FULLTEXT-353

Winkler, M. I., Pasmanik, D., Alvear, K. \& Olivares, B. (2015). La dimensión ética en psicología comunitaria: orientaciones específicas para la práctica y la formación. Santiago, Chile: Universidad de Santiago de Chile.

Yañez Canal, J. (2005). Competencias profesionales del psicólogo clínico: un análisis preliminar. Terapia Psicológica, 23(2), 85-93. Extraído de https://www.redalyc.org/pdf/785/78523209.pdf

Fecha de recepción: Junio de 2017.

Fecha de aceptación: Marzo de 2020. 